\title{
Analysis of Influencing Factors and Pharmaceutical Care of Patients with COVID-19 in Fangcang Shelter Hospital
}

This article was published in the following Dove Press journal: Infection and Drug Resistance

\author{
Fang Cheng ${ }^{1,2}$ \\ Qiang $\mathrm{Li}^{1,2}$ \\ Yong Han ${ }^{1,2}$ \\ Chen Shi ${ }^{1,2}$ \\ Sanlan $\mathrm{Wu}^{1,2}$ \\ Qiling $X u^{1,2}$ \\ Fang Zeng ${ }^{1,2}$ \\ Yu Zhang ${ }^{1,2}$
}

'Department of Pharmacy, Union Hospital, Tongji Medical College, Huazhong University of Science and Technology, Wuhan 430022, People's Republic of China; ${ }^{2}$ Hubei Province Clinical Research Center for Precision Medicine for Critical Illness, Wuhan 430022, People's Republic of China
Correspondence: Fang Zeng; Yu Zhang Department of Pharmacy, Union Hospital, Tongji Medical College, Huazhong

University of Science and Technology,

Wuhan 430022, People's Republic of

China

Email fancyzeng@126.com;

zhangwkp@I63.com
Purpose: In December 2019, coronavirus disease 2019 (COVID-19) was first identified in Wuhan, and rapidly spread throughout China. Patients with mild symptoms were admitted to Fangcang shelter hospitals for centralized quarantine. We aimed to clarify the medication usage, related adverse reactions, and pharmaceutical interventions in patients with mild COVID-19.

Patients and Methods: We innovatively carried out targeted pharmacy services. We provided online and off-line pharmaceutical services to patients in the Fangcang shelter hospital. The use of medication, related adverse reactions, and the effects of pharmaceutical intervention were analyzed.

Results: Lower blood lymphocyte count was proposed as the most significant risk factor in patients with mild illness. All patients received antiviral treatment (arbidol, oseltamivir, and ribavirin); $78.4 \%$ of patients received antibiotic therapy (moxifloxacin, levofloxacin, and cefdinir); patients in the moderate disease group received more antibiotic therapy than those in the mild disease group. Most of the patients were treated with traditional Chinese medicine. Patients with moderate disease preferred to receive sedative hypnotic therapy. Diarrhea, nausea and vomiting, insomnia, arrhythmia, and constipation were the most common adverse reactions. The rate of mild-to-moderate illness in the pharmaceutical intervention and non-intervention groups was $20.6 \%$ and $31.7 \%$, respectively.

Conclusion: Most patients with mild illness were treated with antiviral, antibiotic, and Chinese medicine therapy. However, attention should be paid to patients with mild illness presenting with hypertension and low lymphocyte count at the onset; these patients are more likely to develop moderate or severe disease. Moreover, there were many drug-related problems in Fangcang shelter hospital; pharmaceutical care might contribute to alleviate the progress of the patient's condition. Pharmacists should be prepared to provide skilled and effective services to patients, with the aim to ensure medication safety and promote the overall control of the COVID-19 pandemic.

Keywords: COVID-19, Fangcang shelter hospital, medication, drug-related adverse reactions, pharmaceutical care

\section{Introduction}

In December 2019, coronavirus disease 2019 (COVID-19) was spread in Wuhan, Hubei Province, China; since then, the number of patients has been increasing rapidly. ${ }^{1,2}$ The shortage of medical resources resulted in a large number of patients failing to receive timely treatment, thereby leading to a wider spread of the epidemic. The central steering group made the decision to build Fangcang shelter 
hospitals, in an effort to curb the spread of the epidemic. Patients with mild illness were admitted to the Fangcang shelter hospitals for centralized isolation and observation. This was a major public health measure taken for the particular epidemic period in China. ${ }^{3}$

Jianghan shelter hospital, the first and largest shelter hospital in Wuhan (with nearly 1600 beds in 77 wards), officially received patients on February 5, 2020, and have been operating at full capacity. Owing to the large number of patients and relatively poor medical resources, the diagnosis and treatment activities were difficult under the hospitalization conditions, negatively affecting the timely and effective management of patients. Pharmacists performed pharmaceutical services in Jianghan shelter hospital to compensate for the work gap of medical staff, and provide continuous medication guidance and care for patients. Through offline and online pharmaceutical care, we recorded the medication problems of patients and communicated with doctors for intervention. A total of 448 patients received pharmaceutical care. Data on disease symptoms, examination, medication, and outcome were collected from 116 of those patients.

Most patients had mild COVID-19. Patients with mild illness were encouraged to self-isolate at home in the early stages of the disease. Considering the high infectivity of COVID-19, they were transferred to the Fangcang shelter hospital for centralized isolation and treatment of symptoms. Generally, patients could be discharged after isolation treatment in the shelter hospital following a period of time. However, some patients who deteriorated to moderate or severe illness were transferred to designated hospitals for further treatment. Patients in Fangcang shelter hospital receive medication on their own, leading to many problems due to lack of relevant knowledge. Thus, it was necessary for pharmacists to intervene in patients' medication. This included the screening of patients with potentially severe illness to provide more targeted and effective medical services. At present, most research studies are focused on severe cases who received treatment in the intensive care unit or expired due to the disease. ${ }^{4-6}$ However, by prioritizing patients with mild illness who were not initially suitable for home or Fangcang shelter isolation treatment, we may be able to assist in their recovery and reduce the mortality rate related to the disease.

The medical treatment of patients with mild illness included antiviral medications, and medications targeting comorbidities and complications; adverse reactions and patient compliance have not been reported. These data and experiences are of great significance to the medication management of patients with mild disease in all countries. The objective of this study was to describe the clinical characteristics of 116 patients in Fangcang shelter hospital, and compare mild cases who improved rapidly after treatment with those who progressed to moderate disease. When medical resources are scarce, identifying mild cases through pharmaceutical care is an effective approach. At the same time, we analyzed the clinical medication, adverse reactions, and the effects of pharmaceutical intervention, providing a reference for the clinical treatment of patients with mild COVID-19.

\section{Patients and Methods}

\section{Patient Selection}

This investigation was a retrospective single-center study, which included 116 patients with COVID-19 treated in Jianghan cabin hospital from February 12, 2020 to March 8, 2020. All patients with COVID-19 enrolled in this study were diagnosed according to the World Health Organization interim guidance. According to the severity of the disease, the patients were divided into two groups. The mild disease group included patients who recovered well and were discharged smoothly. The moderate disease group included patients whose disease progression could not be controlled, and were transferred to a designated hospital for further treatment. This case series was approved by the institutional Ethics Board of Union Hospital, Tongji Medical College, Huazhong University of Science and Technology (Wuhan, China) (IRB No. 105). The patient consent was verbal informed consent, and that this was approved by the Institutional Ethics Board of Union Hospital, Tongji Medical College, Huazhong University of Science and Technology. This investigation did not interfere with patient diagnosis or the treatment process.

\section{Data Collection}

Prior to providing online pharmaceutical care, we produced a questionnaire to understand the basic information of patients. We attached the flow chart and questionnaire in the Supplementary Materials (Figure S1 and Table S1). Information obtained through this questionnaire included, demographic data, symptoms, underlying comorbidities, blood tests, clinical medication, and adverse reactions. 
The date of disease onset was defined as the day when the initial symptoms were noticed.

\section{Statistical Analysis}

Categorical variables were described as frequency rates and percentages. Proportions for categorical variables were compared using the $\chi^{2}$ test; the Fisher's exact test was used when the data were limited. When the data were normally distributed, means for continuous variables were compared using independent group $\mathrm{t}$ tests and described using mean \pm standard deviation. Otherwise, the MannWhitney test was performed and data were presented as the median and interquartile range values.

Binary logistic regression analysis was used to predict the presence or absence of any event (dependent variable, "Y") by other variables (independent or explanatory variables, " $\chi_{1}, \ldots, \chi_{\mathrm{p}}$ "). The dependent variable is a discrete variable (dichotomous, with dummy variables coded 0 or 1 , where 0 is either the presence or absence of the event and 1 is the opposite). The independent variables may be continuous or discrete (with dummy variables).

In this study, the binary logistic regression was used to select independent risk factors that affect outcomes. The dependent variable $(\mathrm{Y})$ is severity of illness. Based on this study, the severity of illness was divided into two $(j=2)$ categories (mild and moderate), which has the value corresponding to the level of disease severity $\mathrm{j}$ where $\mathrm{j}=0 ; 1$. The independent variables (age, hypertension, diabetes, dyspnea, diarrhea, sleep quality, blood lymphocyte count, and high-sensitivity C-reaction protein [hsCRP]) are denoted as $\chi_{1}, \chi_{2}, \ldots, \chi_{\mathrm{p}}$. For $\mathrm{j}=0 ; 1$, the binary logistic model was as follows:

$$
\pi(\chi)=\frac{e^{g(\chi)}}{1+e^{g(\chi)}}
$$

or

$$
\begin{aligned}
g(\chi)= & \ln \frac{\pi(\chi)}{1-\pi(\chi)}=\beta_{0}+\beta_{1} \chi_{1}+\beta_{2} \chi_{2} \\
& +\beta_{3} \chi_{3}+\ldots+\beta_{n} \chi_{n}
\end{aligned}
$$

where $\chi=\left(\chi_{1}, \chi_{2}, \ldots, \chi_{p}\right)$ is the vector of predictors for observation, $\beta$ is the corresponding vector of regression coefficients, and $\beta_{0}$ is the intercept. The odds ratios with 95\% confidence intervals were reported for all significant factors to demonstrate the relative odds of the occurrence of the outcome of interest. P-values $<0.05$ denoted statistically significant differences. All statistical analyses were performed using the SPSS version 20.0 software (IBM Corp., Armonk, NY, USA).

\section{Results}

\section{Patient Characteristics}

Of the 116 patients with COVID-19 included in this study, 49 (42.2\%) were males; 87 and 29 patients were diagnosed with mild (mild group) and moderate (severe group) disease, respectively. The median ages of the two groups (mild disease group: 42 years [range: 22-61 years]; moderate disease group: 50 years [range: 32-67 years]) were statistically different. Of the 116 patients, $38(32.8 \%)$ had $\geq 1$ pre-existing conditions. Hypertension ( $\mathrm{N}=15$ [12.9\%]), diabetes $(\mathrm{N}=11[9.5 \%])$, hyperlipemia $(\mathrm{N}=10$ [8.6\%]), and coronary heart disease $(\mathrm{N}=6[5.2 \%])$ were the most common pre-existing conditions. There was no significant difference in hyperlipemia and coronary heart disease between the two groups; however, there was a greater number of patients with hypertension and diabetes in the moderate group than in the mild group (Table 1).

The most common symptoms of patients in the Fangcang shelter hospital were fever $(\mathrm{N}=100[86.2 \%])$, fatigue $(\mathrm{N}=62$ [53.5\%]), cough $(\mathrm{N}=68[58.6 \%])$, sputum production $(\mathrm{N}=33$ [28.5\%]), myalgia ( $N=66[56.9 \%])$, mild shortness of breath $(\mathrm{N}=41$ [35.3\%]), and dyspnea $(\mathrm{N}=29$ [25.0\%]). Less common symptoms included headache, nausea, vomiting, and conjunctivitis (Table 1). A total of 48 patients (41.4\%) had diarrhea. Compared with the mild disease group, patients in the moderate disease group were more likely to report dyspnea and diarrhea. In addition, 65 (56.0\%) and 21 (18.1\%) patients reported a sleep problem and anorexia, respectively.

The lymphocyte count of most patients was normal or slightly lower, while the eosinophil count was generally low. There was no significant difference in the number of white blood cells, neutrophils, eosinophils, and platelets, as well as the level of hemoglobin, between the two groups. Nevertheless, in patients with moderate illness, the blood lymphocyte count was significantly lower, and hsCRP was significantly increased. A negative polymerase chain reaction result is one of the prerequisites for release from quarantine. The median time of the two groups was statistically different: $(16.7 \pm 6.2)$ and $(25.2 \pm 9.7)$ days in the mild and moderate disease groups, respectively (Table 2).

\section{Prognostic Factors of Moderate Illness}

A total of 23 variables were included in the univariate analysis. The results showed that age, hypertension, 
Table I Demographic and Clinical Characteristics of II6 Patients with COVID-I9 Pneumonia

\begin{tabular}{|c|c|c|c|c|}
\hline & $\mathbf{N}(\%)$ & & & \\
\hline & Total $(\mathrm{N}=1 \mid 6)$ & Mild $(\mathrm{N}=87)$ & Moderate $(\mathrm{N}=29)$ & $P$-value ${ }^{a}$ \\
\hline Age, median (range), years & $44(22-67)$ & $42(22-6 I)$ & $50(32-67)$ & 0.008 \\
\hline \multicolumn{5}{|l|}{ Sex } \\
\hline Female & $49(42.2)$ & $37(42.5)$ & $12(4 \mid .4)$ & 0.914 \\
\hline Male & $67(57.8)$ & $50(57.5)$ & $17(58.6)$ & \\
\hline \multicolumn{5}{|l|}{ Comorbidity } \\
\hline Hypertension & $15(12.9)$ & $6(6.9)$ & $9(31.0)$ & 0.001 \\
\hline Diabetes & II (9.5) & $5(5.7)$ & $6(20.7)$ & 0.017 \\
\hline Hyperlipemia & $10(8.6)$ & $7(8.0)$ & $3(10.3)$ & 0.702 \\
\hline Coronary heart disease & $6(5.2)$ & $3(3.4)$ & $3(10.3)$ & 0.146 \\
\hline Others & $9(7.8)$ & $6(6.9)$ & $3(10.3)$ & 0.548 \\
\hline \multicolumn{5}{|l|}{ Sign and symptom } \\
\hline Fever & $100(86.2)$ & $75(86.2)$ & $25(86.2)$ & 0.880 \\
\hline Dry cough & $68(58.6)$ & $53(60.6)$ & $15(51.7)$ & 0.384 \\
\hline Sputum production & $33(28.5)$ & $26(30.0)$ & $7(24.1)$ & 0.552 \\
\hline Fatigue & $62(53.5)$ & $48(55.2)$ & $14(48.3)$ & 0.294 \\
\hline Shortness of breath & $4 \mathrm{I}(35.3)$ & $32(36.8)$ & $9(31.0)$ & 0.575 \\
\hline Myalgia & $66(56.9)$ & $47(54.0)$ & $19(65.5)$ & 0.279 \\
\hline Dyspnea & $29(25.0)$ & $17(19.5)$ & $12(4 \mid .3)$ & 0.019 \\
\hline Diarrhea & $48(4 I .4)$ & $31(35.6)$ & $17(58.6)$ & 0.029 \\
\hline Others & $35(30.2)$ & $26(30.0)$ & $9(31.0)$ & 0.907 \\
\hline \multicolumn{5}{|l|}{ Quality of life } \\
\hline Sleep status ${ }^{b}$ & $65(56.0)$ & $44(51.6)$ & 21 (72.4) & 0.040 \\
\hline Dietary status $^{c}$ & $21(18.1)$ & $15(17.2)$ & $6(23.1)$ & 0.427 \\
\hline
\end{tabular}

Notes: ${ }^{\mathrm{a}} \mathrm{P}$-values indicate differences between patients with mild and moderate symptoms. $\mathrm{P}<0.05$ denotes statistically significant difference. ${ }^{\mathrm{b}}$ Sleep status refers to poor sleep quality. 'Dietary status refers to poor dietary quality.

Abbreviations: N, number; COVID-19, coronavirus disease 2019.

diabetes, dyspnea, diarrhea, sleep quality, blood lymphocyte count, and hsCRP obtained from the 116 cases were predictive factors for the incidence of severe illness. The aforementioned eight factors were included in the logistic regression analyses. The results showed that blood lymphocyte count was the most useful prognostic factors affecting the prognosis (Table 3). Additionally, we conducted a collinearity analysis between the included variables in the Supplementary Materials (Table S2A and B). The results showed that the variance inflation factor (VIF) were all less than 10 and tolerance values all more than 0.2 , indicating that there was no collinearity between the variables.

\section{Medication}

According to the medication use of the 116 patients, the patients generally received treatment with antiviral, antibiotic, antitussive, traditional Chinese medicine (including
Lianhua qingwen capsule and traditional Chinese medicine decoction), and sedative hypnotic drugs (Table 4).

The majority of patients in the Fangcang field hospital were treated with oral medication. All 116 patients received antiviral treatment; of those, 74 patients $(63.8 \%)$ received arbidol tablets (200 mg every $8 \mathrm{~h}$, orally), 100 patients (86.2\%) self-administered oseltamivir in the early stages of onset (75 mg twice daily, orally), and six patients (5.2\%) received ribavirin injection at fever clinics. A total of 91 patients (78.4\%) received antibiotic (moxifloxacin, levofloxacin, cefdinir) therapy; in the moderate disease group, $91.3 \%$ received antibiotic therapy. Most patients were treated with traditional Chinese medicine. 110 patients $(94.8 \%)$ received traditional Chinese medicine decoction (twice daily, orally), and Lianhua qingwen capsule (1400 mg every $8 \mathrm{~h}$, orally) for consolidation therapy. Some patients with severe cough symptoms received antitussive therapy. A small number of patients with mild disease required sedative hypnotics, while 
Table 2 Laboratory Findings of 116 Patients with COVID-19 Pneumonia

\begin{tabular}{|c|c|c|c|c|c|}
\hline & Normal Range & Total $(\mathrm{N}=|| 6)$ & Mild $(\mathrm{N}=87)$ & Moderate $(\mathrm{N}=29)$ & P-value ${ }^{a}$ \\
\hline White blood cell count, $\times 10^{9} / \mathrm{L}$ & $3.5-9.5$ & $5.4(4.1-7.0)$ & $5.6(4.6-7.1)$ & $4.9(3.5-6.3)$ & 0.124 \\
\hline Neutrophil count, $\times 10^{9} / \mathrm{L}$ & $1.8-6.3$ & $3.5(2.5-4.3)$ & $3.7(2.6-4.4)$ & $3.1(2.3-3.8)$ & 0.413 \\
\hline Lymphocyte count, $\times 10^{9} / \mathrm{L}$ & $1.1-3.2$ & $1.3 \pm 0.6$ & $1.5 \pm 0.6$ & $0.9 \pm 0.3$ & $<0.001$ \\
\hline Eosinophils, $\times 10^{9} / \mathrm{L}$ & $0.02-0.52$ & $0.01(0.00-0.02)$ & $0.01(0.00-0.03)$ & $0.01(0.01-0.03)$ & 0.117 \\
\hline Hemoglobin, g/L & $115-150$ & $142(132-153)$ & $143(\mid 34-153.75)$ & $139(124-151.00)$ & 0.402 \\
\hline Platelet count, $\times 10^{9} / \mathrm{L}$ & $125-350$ & $197(150-245)$ & $209.5(152.5-243.0)$ & $179(135.0-289.0)$ & 0.769 \\
\hline hsCRP, mg/L & $<4.0$ & $6(0.9-20.1)$ & $2.6(0.6-11.5)$ & $17.4(6.8-45.7)$ & $<0.001$ \\
\hline Virus clearance time $^{\mathrm{b}}$ (days) & & $18.9 \pm 8.1$ & $16.7 \pm 6.2$ & $25.2 \pm 9.7$ & $<0.001$ \\
\hline
\end{tabular}

Notes: ${ }^{\text {P }}$-values indicate differences between patients with mild and moderate symptoms. $\mathrm{P}<0.05$ denotes statistically significant difference. ${ }^{b}$ Virus clearance time is from the day of positive detection of the nucleic acid to the time when the test was negative (twice).

Abbreviations: N, number; COVID-19, coronavirus disease 2019; hsCRP, high-sensitivity C-reaction protein.

Table 3 Logistic Regression Analyses of Risk Factors Associated with Mild-to-Moderate Disease

\begin{tabular}{|l|l|l|l|}
\hline & OR & 95\% CI & P-value $^{\mathbf{a}}$ \\
\hline Age & 0.97 & $0.89-1.06$ & 0.496 \\
Hypertension & 0.09 & $0.01-0.72$ & 0.053 \\
Diabetes & 0.14 & $0.01-1.99$ & 0.146 \\
Dyspnea & 0.77 & $0.08-1.33$ & 0.117 \\
Diarrhea & 0.95 & $0.21-4.37$ & 0.952 \\
Sleep status & 0.31 & $0.06-1.66$ & 0.171 \\
Lymphocyte count $^{\text {bympr }}$ & 0.01 & $0-0.14$ & 0.001 \\
hsCRP & 1.007 & $0.98-1.03$ & 0.569 \\
\hline
\end{tabular}

Notes: ${ }^{\text {a }}$-values indicate differences between patients with mild and moderate symptoms. $P<0.05$ denotes statistically significant difference. ${ }^{b}$ Sleep status refers to poor sleep quality.

Abbreviations: $\mathrm{OR}$, odds ratio; $\mathrm{Cl}$, confidence interval; hsCRP, high-sensitivity C-reaction protein.

most patients with moderate illness needed drugs to maintain sleep quality.

\section{Drug-Related Adverse Reactions}

Patients in the Fangcang shelter hospital received multiple medication regimens. Of note, $32.8 \%$ of the 116 patients had $\geq 1$ coexisting medical conditions, which markedly increased the complexity of the medication use and incidence of adverse reactions. Of the 116 patients, 80 (69.0\%) experienced $\geq 1$ adverse reactions. Diarrhea $(\mathrm{N}=40$ [34.5\%]), nausea and vomiting $(\mathrm{N}=38$ [32.8\%]), insomnia $(\mathrm{N}=20[17.2 \%])$, arrhythmia $(\mathrm{N}=16[13.8 \%])$, and constipation $(8[6.9 \%])$ were the most common adverse reactions (Table 5).

\section{Pharmaceutical Intervention}

We designed a questionnaire for discharged patients to evaluate the patient satisfaction and effect of pharmaceutical care (Table S3). The results of the pharmaceutical intervention are shown in Tables $6-8$. We provided 758 online pharmaceutical care services to the 116 patients. The pharmaceutical care service included information regarding infection and protection from COVID-19 (30.2\%), drug-related counseling (56.3\%), environmental problems at Fangcang hospital (2.0\%), psychological counseling (10.2\%), and others (1.3\%). The drug-related problems included inappropriate combination of drugs (13.6\%), dosage $(6.2 \%)$, course of antibiotic and antiviral treatment (48.4\%), drug interaction problems (5.5\%), and adverse reactions (26.3\%). Most of the proposed advice or explanations provided by a clinical pharmacist were accepted by patients (Table 6).

We compared the rate of mild-to-moderate illness between the pharmaceutical intervention and nonintervention groups (Table 7). The results showed that this rate was $20.6 \%$ and $31.7 \%$, respectively, indicating that pharmaceutical care may be associated with a decreased rate of mild-to-moderate illness.

According to the feedback, most patients responded that pharmaceutical care contributed to their recovery, and they were satisfied with the pharmaceutical care service $(62.9 \%$ and $21.6 \%$ were very satisfied and somewhat satisfied, respectively) (Table 8).

\section{Discussion}

In view of the rapid human-to-human transmission of COVID-19, ${ }^{7,8}$ Fangcang shelter hospitals were established to quarantine patients with mild illness. In this study, the data of 116 patients with mild and moderate COVID-19 were analyzed, and their baseline characteristics were described and compared. Subsequently, the clinical medication and drug-related adverse reactions were reviewed.

Previous studies have found that severe disease or death due to COVID-19 occurred mostly in older 
Table 4 Clinical Medication of II6 Patients with COVID-19 Pneumonia

\begin{tabular}{|c|c|c|c|c|}
\hline & N (\%) & & & \\
\hline & $\begin{array}{l}\text { Total } \\
(\mathrm{N}=|| 6)\end{array}$ & $\begin{array}{l}\text { Mild } \\
(\mathrm{N}=87)\end{array}$ & $\begin{array}{l}\text { Moderate } \\
(\mathrm{N}=29)\end{array}$ & P-value ${ }^{a}$ \\
\hline \multicolumn{5}{|l|}{ Antiviral } \\
\hline Arbidol & $74(63.8)$ & $55(63.2)$ & $19(65.5)$ & 0.823 \\
\hline Oseltamivir & $100(86.2)$ & $77(88.5)$ & $23(79.3)$ & 0.214 \\
\hline Ribavirin $^{\mathrm{b}}$ & $6(5.2)$ & $5(5.7)$ & I (3.4) & 0.628 \\
\hline Antibiotic (moxifloxacin, levofloxacin, cefdinir) & 91 (78.4) & $64(73.6)$ & 27 (93.I) & 0.026 \\
\hline \multicolumn{5}{|l|}{ Traditional Chinese medicine } \\
\hline Lianhua qingwen capsule & $110(94.8)$ & $83(95.4)$ & $27(93.1)$ & 0.841 \\
\hline Traditional Chinese medicine decoction & $110(94.8)$ & $83(95.4)$ & $27(93.1)$ & $0.84 I$ \\
\hline $\begin{array}{l}\text { Antitussives (Suhuang Zhike capsule, compound methoxyphenamine } \\
\text { capsule) }\end{array}$ & $37(31.9)$ & $27(31.0)$ & $10(34.5)$ & 0.73 \\
\hline Sedative hypnotic therapy (alprazolam) & $44(37.9)$ & $19(16.4)$ & $25(86.2)$ & $<0.001$ \\
\hline
\end{tabular}

Notes: ap-values indicate differences between patients with mild and moderate. $\mathrm{P}<0.05$ denotes statistically significant difference. ${ }^{\mathrm{b}} \mathrm{Patients}$ in the Fangcang shelter hospital almost treated with oral administration. Patients received ribavirin injection at fever clinics in the early stages of COVID-19.

Abbreviations: N, number; COVID-19, coronavirus disease 2019.

Table 5 Drug-Related Adverse Reactions of II6 Patients with COVID-19 Pneumonia

\begin{tabular}{|l|l|l|l|}
\hline & $\mathbf{N}(\%)$ & & \\
\cline { 2 - 4 } & $\begin{array}{l}\text { Total } \\
\text { (N=I I6) }\end{array}$ & $\begin{array}{l}\text { Mild type } \\
\mathbf{( N = 8 7 )}\end{array}$ & $\begin{array}{l}\text { Moderate type } \\
\text { (N=29) }\end{array}$ \\
\hline $\begin{array}{l}\text { Diarrhea } \\
\text { Nausea and }\end{array}$ & $40(34.5)$ & $29(33.3)$ & II (37.9) \\
vomiting & $38(32.8)$ & $29(33.3)$ & $9(31.0)$ \\
Insomnia & $20(17.2)$ & $15(17.2)$ & $5(17.2)$ \\
Arrhythmia & $16(13.8)$ & $11(12.6)$ & $5(17.2)$ \\
Constipation & $8(6.9)$ & $4(4.6)$ & $4(13.8)$ \\
Others & $13(11.2)$ & $9(10.3)$ & $4(13.8)$ \\
\hline
\end{tabular}

Abbreviations: N, number; COVID-19, coronavirus disease 2019.

individuals with comorbidities. ${ }^{4,9}$ In this study, the median age of patients with mild and moderate illness was 42 and 50 years, respectively. The patients in the moderate disease group had more comorbidities than those in the mild disease group, mainly including hypertension, diabetes, and coronary heart disease. However, there was no significant difference between patients with mild and moderate illness.

Common symptoms in patients with mild illness at the onset were fever, cough, mild shortness of breath, myalgia, and fatigue, while some developed dyspnea. However, a significant proportion of patients presented with gastrointestinal and ocular symptoms, such as diarrhea, nausea, and conjunctivitis. Notably, there was difference in the incidence of diarrhea and dyspnea between patients with mild and moderate illness, which may be related to poor outcomes. The onset of symptoms may assist doctors in identifying patients with poor prognosis. ${ }^{10,11}$

Patients with moderate symptoms at the onset of illness presented higher hsCRP levels than those in the mild disease group, indicating that inflammation may affect disease progression. Moreover, patients with moderate illness were more likely to be infected by bacteria. The decrease in the lymphocyte count was related to the progression of disease. The lower blood lymphocyte count may be a potential indicator for diagnosis in the future. Patients with moderate illness have longer virus clearance time, which was also consistent with disease progression.

Thus far, there is no targeted treatment for COVID-19, except for supportive care to patients. According to the medication data of the 116 patients, most patients received treatment with antiviral agents. Oseltamivir and antibacterial agents are ineffective against COVID-19. ${ }^{12}$ However, the early stage of the disease happened to be the high incidence of influenza every year; patients usually receive oseltamivir for the treatment of common influenza. Indeed, some of the 116 patients had signs of bacterial infection at the onset of illness, and they were treated with antibiotics. However, the diagnosis and treatment plan for COVID-19 issued by the National Health and Health Commission recommended that blind or inappropriate use of antibiotics should be avoided, especially the combination of broad-spectrum antibiotics. ${ }^{13}$ Patients in Fangcang shelter hospitals generally accepted treatment 
Table 6 Service Content of Pharmaceutical Intervention

\begin{tabular}{|l|l|l|}
\hline $\begin{array}{l}\text { Pharmaceutical Care } \\
\text { Service }\end{array}$ & N (\%) \\
\hline $\begin{array}{l}\text { Infection and protection } \\
\text { of COVID-19 }\end{array}$ & $229(30.2)$ \\
\hline & $\begin{array}{l}\text { Inappropriate } \\
\text { combination of drugs }\end{array}$ & $58(13.6)$ \\
\hline Drug related counseling & $\begin{array}{l}\text { Dreatment course of } \\
\text { antibiotic and antiviral }\end{array}$ & $207(48.4)$ \\
\hline $\begin{array}{l}\text { Environmental problems } \\
\text { in Fangcang hospital }\end{array}$ & $\begin{array}{l}\text { Drug interaction } \\
\text { problems }\end{array}$ & $23(5.4)$ \\
\hline $\begin{array}{l}\text { Psychological counseling } \\
\text { Others }\end{array}$ & Adverse reactions & $113(26.4)$ \\
\hline
\end{tabular}

Table 7 Effect of Pharmacist Intervention on Patients with Mildto-Moderate COVID-19

\begin{tabular}{|l|l|}
\hline & Mild-to-Moderate Rate (\%) \\
\hline Intervention & 20.6 \\
Non-intervention & 31.7 \\
\hline
\end{tabular}

Abbreviation: COVID-19, coronavirus disease 2019.

Table 8 Patient Satisfaction of Pharmaceutical Intervention

\begin{tabular}{|l|l|}
\hline Patient Satisfaction Score & N (\%) \\
\hline 5: Very Satisfied & $73(62.9)$ \\
4: Somewhat Satisfied & $25(21.6)$ \\
3: Neutral & $8(6.9)$ \\
2: Somewhat Unsatisfied & $6(5.2)$ \\
I: Very Unsatisfied & $4(3.4)$ \\
Mean (SD) of Scores & $4.4(1.17)$ \\
\hline
\end{tabular}

with traditional Chinese medicine, which showed great advantages in the prevention and control of the epidemic. ${ }^{14}$ They were generally more inclined to treatment with traditional Chinese medicine during the recovery period.

Patients in the Fangcang shelter hospital suffered from certain psychological problems, mainly manifested as sadness, complaints, excessive anxiety, and insomnia, which were not conducive to treatment and recovery. ${ }^{15}$ Alprazolam was often used to relieve insomnia. Meanwhile, patients should receive sufficient nutritional support to improve their immunity, and maintain a regular internal clock to ensure adequate sleep. The pharmacists need to think about the transposition, as well as timely and patiently provide medication and treatment services to patients, ${ }^{16}$ to ensure an efficient diagnosis and treatment in the Fangcang shelter hospital. Furthermore, pharmacists could provide medical science communication for patients to stabilize their psychological status.

In terms of treatment safety and tolerability, the use of moxifloxacin was associated with the occurrence of serious gastrointestinal adverse reactions and anorexia. A large number of patients experienced panic and arrhythmia after receiving oseltamivir or arbidol. It has been reported that COVID-19 may invade and cause damage to the heart, kidneys, and other organs through angiotensin I converting enzyme 2 (ACE2). ${ }^{17,18}$ Patients with hypertension and heart disease received cautious treatment with antitussives, such as Suhuang Zhike capsule and compound methoxyphenamine capsule. When moxifloxacin and levofloxacin are administered in patients with diabetes, physicians should carefully monitor the levels of blood sugar. There were numerous cases of gastrointestinal tract discomfort caused by the Lianhua qingwen capsule, and diarrhea caused by the combination with traditional Chinese medicine decoction. We also found that patients with moderate illness had different degrees of liver damage with the prolongation of the treatment process, which may be related to the hepatotoxicity of drugs. Therefore, pharmacists should pay attention to the drugrelated adverse reactions, ${ }^{19}$ including to determine the cause and effect of adverse drug reactions, such as the correlation of medication time.

We collected patient information and data on medication problems (eg, dosage, combined medication, adverse reactions, etc.) through pharmacy services. The results showed that timely intervention by pharmacists can significantly improve the disease progression and contribute to a swift recovery from illness and discharge from hospital.

\section{Conclusion}

There were some limitations in the study. First, this was a single-center study with a small sample. Second, data on laboratory parameters of patients in the Fangcang shelter hospital were limited, and their acquisition may be delayed due to actual conditions. Moreover, we did not follow up on the clinical outcome of some patients with moderate illness. However, we focused on the clinical medication 
and related adverse reactions in patients with mild disease from the perspective of pharmaceutical intervention. Most patients with mild illness were treated antiviral agents, antibiotics, and Chinese medicine therapy. However, attention should be paid to patients with mild illness accompanied by low lymphocyte count at the onset, who were more likely to develop moderate or severe disease. Moreover, pharmaceutical care could markedly reduce the risk of mild-to-moderate illness. According to the feedback, most patients responded that pharmaceutical care contributed to their recovery. Therefore, pharmacists should be prepared to provide skilled and effective services to patients, with the aim to ensure medication safety and promote the overall control of the COVID-19 pandemic.

\section{Funding}

This work was supported by the National Key R\&D Program of China (2017YFC0909900).

\section{Disclosure}

All authors report no conflicts of interest relevant to this article.

\section{References}

1. Li Q, Guan X, Wu P, et al. Early transmission dynamics in Wuhan, China, of novel coronavirus-infected pneumonia. $N$ Engl $J$ Med. 2020;382:1199-1207. doi:10.1056/NEJMoa2001316

2. Phelan AL, Katz R, Gostin LO. The novel coronavirus originating in Wuhan, China: challenges for global health governance. JAMA. 2020;323(8):709-710. doi:10.1001/jama.2020.1097

3. Chen S, Zhang Z, Yang J, et al. Fangcang shelter hospitals: a novel concept for responding to public health emergencies. Lancet. 2020;395 (10232):1305-1314. doi:10.1016/S0140-6736(20)30744-3

4. Zhou F, Yu T, Du R, et al. Clinical course and risk factors for mortality of adult inpatients with COVID-19 in Wuhan, China: a retrospective cohort study. Lancet. 2020;395(10229):1054-1062. doi:10.1016/ S0140-6736(20)30566-3

5. Wang D, Hu B, Hu C, et al. Clinical characteristics of 138 hospitalized patients with 2019 novel coronavirus-infected pneumonia in Wuhan, China. JAMA. 2020;323(11):1061-1069. doi:10.1001/jama.2020.1585
6. Liu J, Liu Y, Xiang P, et al. Neutrophil-to-lymphocyte ratio predicts severe illness patients with 2019 novel coronavirus in the early stage. $J$ Transl Med. 2020;18(1):206. doi:10.1186/s12967-020-02374-0

7. Chan J, Yuan S, Kok KH, et al. A familial cluster of pneumonia associated with the 2019 novel coronavirus indicating person-toperson transmission: a study of a family cluster. Lancet. 2020;395 (10223):514-523. doi:10.1016/S0140-6736(20)30154-9

8. Yu P, Zhu J, Zhang Z, et al. A familial cluster of infection associated with the 2019 novel coronavirus indicating possible person-to-person transmission during the incubation period. $J$ Infect Dis. 2020;221 (11):1757-1761. doi:10.1093/infdis/jiaa077

9. Yang X, Yu Y, Xu J, et al. Clinical course and outcomes of critically ill patients with SARS-CoV-2 pneumonia in Wuhan, China: a single-centered, retrospective, observational study. Lancet Respir Med. 2020;8(5):475-481. doi:10.1016/S2213-2600(20)30079-5

10. Chen N, Zhou M, Dong X, et al. Epidemiological and clinical characteristics of 99 cases of 2019 novel coronavirus pneumonia in Wuhan, China: a descriptive study. Lancet. 2020;395 (10223):507-513. doi:10.1016/S0140-6736(20)30211-7

11. Mo P, Xing Y, Deng L, et al. Clinical characteristics of refractory COVID-19 pneumonia in Wuhan, China. Clin Infect Dis. 2020. doi:10.1093/cid/ciaa270

12. National Health Commission of the People's Republic of China. Chinese management guideline for COVID-19 (version 7.0); 2020. Available from: http://www.nhc.gov.cn/yzygj/s7653p/202002/ 8334 a 8326 dd 94 d 329 df 351 d 7 da 8 aefc $2 /$ files/b 218 cfeb 1 b c54639af227f922bf6b817.pdf. Accessed September 11, 2020.

13. Jin Y, Cai L, Cheng Z, et al. A rapid advice guideline for the diagnosis and treatment of 2019 novel coronavirus $(2019-\mathrm{nCoV})$ infected pneumonia (standard version). Mil Med Res. 2020;7:4.

14. Du H, Hou X, Miao Y, et al. Traditional Chinese medicine: an effective treatment for 2019 novel coronavirus pneumonia (NCP). Chin J Nat Med. 2020;18:206-210.

15. Nguyen HC, Nguyen MH, Do BN, et al. People with suspected COVID-19 symptoms were more likely depressed and had lower health-related quality of life: the potential benefit of health literacy. J Clin Med. 2020;9(4):965. doi:10.3390/jcm9040965

16. Meng L, Qiu F, Sun S. Providing pharmacy services at cabin hospitals at the coronavirus epicenter in China. Int J Clin Pharm. 2020;42 (2):305-308. doi:10.1007/s11096-020-01020-5

17. Zou X, Chen K, Zou J, et al. The single-cell RNA-seq data analysis on the receptor ACE2 expression reveals the potential risk of different human organs vulnerable to Wuhan 2019-nCoV infection. Front Med. 2020;14(2):185-192. doi:10.1007/s11684-020-0754-0

18. Cui Y, Tian M, Huang D, et al. A 55-day-old female infant infected with 2019 novel coronavirus disease: presenting with pneumonia, liver injury, and heart damage. $J$ Infect Dis. 2020;221 (11):1775-1781. doi:10.1093/infdis/jiaa113

19. Cadogan CA, Hughes CM. On the frontline against COVID-19: community pharmacists' contribution during a public health crisis. Res Social Adm Pharm. 2020. doi:10.1016/j.sapharm.2020.03.015
Infection and Drug Resistance

\section{Publish your work in this journal}

Infection and Drug Resistance is an international, peer-reviewed openaccess journal that focuses on the optimal treatment of infection (bacterial, fungal and viral) and the development and institution of preventive strategies to minimize the development and spread of resistance. The journal is specifically concerned with the epidemiology of antibiotic resistance and the mechanisms of resistance development and diffusion in both hospitals and the community. The manuscript management system is completely online and includes a very quick and fair peerreview system, which is all easy to use. Visit http://www.dovepress.com/ testimonials.php to read real quotes from published authors. 